\title{
Analysis about Benefits of Software-Defined Wide Area Network: A New Alternative for WAN Connectivity
}

\author{
Catherine Janiré Mena Diaz, Laberiano Andrade-Arenas, Javier Gustavo Utrilla Arellano, Miguel Angel Cano Lengua \\ Facultad de Ingenieria de Sistemas e Informatica \\ Universidad Tecnológica del Perú \\ Lima, Perú
}

\begin{abstract}
This article is based on conducting research to analyze the benefits of emerging trends in communications and networking technology, such as software-defined wide area networks. Using Waterfall as a methodology, the main objective is to carry out a technical comparison at the design and configuration level, creating a virtual environment that simulates traditional and SDWAN (Software-Defined Wide Area Network) infrastructures. The results obtained verify that the benefits of SDWAN maintain business continuity, anticipate situations in which the infrastructure can act intelligently, optimize connectivity while maintaining security, and provide improvements in the management of the entire infrastructure. People will be able to see the results obtained between both technologies and validate the benefits that SDWAN offers.
\end{abstract}

Keywords-Networking technology; connectivity; SDWAN; wide area network; waterfall

\section{INTRODUCTION}

Nowadays, for many companies, communications are a vital part of the business continuity, however, in this process the infrastructure and technology that carry out this activity goes unnoticed.

All companies that provide services need to maintain an agile data transmission, as well as an infrastructure that supports it. In turn, connectivity between the company's headquarter, branch offices and data center is a very important aspect because it is necessary to provide services to its customers continuously and with high availability. For this reason, most companies opt to use private networks that are deployed and managed by external service providers, however, today a new technology is being used called SDWAN to communicate all the company's sites. This technology provides a significant change on networking because it delivers innovation in the way it operates, adds a return of investment in medium term and several benefits that makes more companies dare to invest in it [1].

This research work aims to present SDWAN as a new technological alternative for WAN connectivity between multiple sites, because traditionally for 15 years it has been using traditional WAN or known as Multiprotocol Label Switching (MPLS) in Peru. MPLS is known in the technological field as the most popular communication protocol and used by service providers to connect multiple locations of their customers. SDWAN is a relatively new technology in the world since it is only about 3 years since it became more widely known and it is being adopted by more companies every year.

It is necessary to emphasize that in Peru there are many companies that do not dare to make technological changes or renovations due to a lack of knowledge or rejection of change. Therefore, this research focuses on the benefits that SDWAN technology can bring, for this purpose, the operational benefits at the deployment level of software-defined network solutions for WAN connectivity are analyzed. In addition to the technical benefits at the scalability level and the economic benefits of the software-defined networking solution. Likewise, as support for the research, laboratory tests are carried out that provide technical results in which the benefits that this technology brings in comparison with traditional WAN technology are manifested, not only at a theoretical level, but also at practical level.

This article has the objective to analyze the benefits at the operational, technical, economic level and the comparison between the traditional technology and SDWAN.

\section{LITERATURE REVIEW}

According to the growing demand of users towards companies for the use of their services; the use of the internet and the need to maintain connectivity have been fundamental factors in order to deploy applications and meet the expectations of its customers. It can be said that any company that provides services needs to maintain an agile data transmission, as well as an infrastructure that supports it. Nowadays companies are not only looking for availability, reliability and performance so that they can provide connectivity between their sites. Now they are also looking for scalability [2]; for that is inevitable to speak of wide area networks (WAN) when it comes to transmitting data or information. In turn, connectivity, between the sites that a company may have, is a very important issue because it is necessary to provide services to its customers continuously and with high availability. Most companies choose to use private networks that are deployed and managed by external service providers. However, today a new technology is being used to communicate the headquarters of a company, called softwaredefined wide area networks. This technology provides a change in the use of traditional networks because it delivers innovation in the way it operates, adds a share of cost and benefit in the medium term that makes more companies dare to invest in it 
[3]. Finally, it removes the prominence of service providers since it provides greater autonomy to clients to be able to manage their networks [1].

One characteristic that SDWAN has is that it uses three planes: the data plane, which contributes to making communication between sites easier, since a logical infrastructure is created which will work on a physical infrastructure and will allow to transfer data from origin to destinations in a fluid way; the control plane will carry out the control of the configurations of the devices connected to the network, such as policies, routing information, accesses [1]; the orchestration plan that provides a business policy and security framework.

One of the advantages in the use of SDWAN in wide area networks, is that it allows the configuration and management of connections between branches of a company is simple and also flexible, easy to control and supervise, which in the medium and long term results in reducing operating costs [4]. This type of network emerge as a solution to face the different deficiencies that traditional networks present [5],because they facilitate bandwidth management and prioritize data traffic in WAN networks that can also be done in traditional networks, but which requires more effort.

Another advantage that SDWAN networks have is the management, control and configuration of the company's networks from a centralized web platform. This allows changes that are applied to different network connections to take effect immediately. In the centralized platform it will be possible to visualize in a unified way parameters such as the data consumption of the interfaces, latencies of the links, use of the bandwidth by IP, the available bandwidth and other statistics that allow optimal monitoring and control of the network status [4]. It also mentions that in traditional wide area networks to be able to update a network successfully, it is usually necessary to configure the devices manually, which is time consuming and prone to errors compared to an SDWAN type network, in which everything is executed from the centralized platform. Likewise, the platform makes it possible to differentiate the types of traffic that are used by clients or by the companies that manage this platform. These types of traffic are: video traffic, voice traffic, data traffic, and management traffic. Each of them has a prioritization over the other which is defined as follows: video over voice, voice over data and data on administration.

Thus, in a comparison of scenarios to measure and verify the effectiveness of software-defined networks, a simulation was carried out, for which they created a virtual scenario and implemented an SDWAN network. Which allows to establish communication between 2 data centers that use softwaredefined network technology and in whose tests voice information was transmitted over IP. As a result of the tests carried out, it was evidenced that by configuring the traffic prioritization policies, it is possible to guarantee that the bandwidth quality of service works at an adequate level, in addition to only using a low percentage of CPU load, which translates into efficient network management [5].

In another investigation, 2 scenarios are compared. The first corresponds to a network configured with classic IP / MPLS using manual routing policies and the second scenario uses a software-defined WAN network, for both scenarios the same number of routers and links are used. In the first scenario, manual routing policies were configured in which low or high latency routes were established. When a router needs to establish communication with another and requests a low latency, the communication will be sent on those links configured with low latency. If at any time the latency of these links increases, the communication will be sent through the same route, as the configuration establishes it. While, in the second scenario, in which a software-defined WAN network is used, the network controller is the one in charge of continuously measuring the status of the links and dynamically defining which route should be used to send the communication [6] , as it is programmed in such a way that it has a complete view of the network topology.

In another investigation similar to the one mentioned in the previous paragraph, they evaluated the performance of implementing a software-defined WAN network in an enterprise. In this investigation, they communicated two branches to their headquarters through two Internet / Broadband connections. In the simulation, 2 performance metrics are considered, which are the service time and the percentage of lost packets. The configuration established as the delay time is $10 \mathrm{~ms}$ and a loss of 2 packets per second (pps). During the simulation, an increase in the delay time was induced, to cause packet losses. When the SDWAN controller detects that the configuration parameters have been exceeded, it automatically establishes a route change for sending the packets, so that it does not affect the quality of service (QoS) [7]. Another investigation refers to the traffic routing associated with applications and administration and how these can be optimized by means of a module added to the controller [8].

Another point to consider in the use of SDWAN technology is that it can work with several connection links, through which traffic is routed through a WAN network making use of load balancing [9], which allows to improve and manage the traffic of the network in the different links [10].

On the other hand, examples of the use of SDWAN in the banking sector are presented. In 2017, there was a drop of 8,800 ATMs in India. Because the satellite connections had a disconnection due to a failure in the satellite leaving many ATMs unusable. This resulted in customers being unable to make transfers or transactions until the failure that cost approximately 600to 900 billion was resolved. As a lesson learned, it was decided to bet on making viable a project in which important points are considered such as having a connection in contingency in case the main link falls and that is cost effective for the banks. In such a way that it adapts to the existing environment at a technical and cost level, as well as the investment and expected performance, appropriate to the services and the architecture [11]. For this reason, a plan was deployed in the cities of Java and Bali (Indonesia) that in a period of 3.5 years provided satisfactory results and in that same period of time many other companies began to invest in wide area networks defined by software.

The example above explains how SDWAN operates in this specific scenario. Provide the feasibility of having a connection as a contingency in case the satellite connection has any failure. The redundant connection can work in an active / active or active / available way, in either case the objective is to keep the WAN connection secure. The secondary or contingency medium that was chosen in this scenario was $4 \mathrm{G} /$ LTE. For 
this reason, SDWAN can benefit companies that have many locations and that need to keep their WAN connection always active, in addition, with the TCAC it is demonstrated in an agile way that indicates feasibility and that it can be cost effective.

It is also important to mention that software-defined wide area networks are a relatively new technology and for that reason many companies have not yet chosen to use it. For example, in India, $75 \%$ of business customers want to start using these kinds of new solutions; however, only 5\% of customers risk making the change, even though it has been shown that this type of network generates great benefits [12], which allows companies to have lower operating costs, higher performance and a robust deployment of software-defined wide area networks. With this technology it can be said that the bottlenecks to transfer information will no longer exist.

Additionally, when comparing traditional WAN networks with SDWAN, the latter are notably superior because in addition to providing centralized control, they allow defining network policies based on profiles and managing data traffic without the need for individual configurations [4]. Also, by massively coupling similar configuration profiles to a set of computers, which would help facilitate the tasks of network administrators because they could manage tasks jointly making more efficient use of work times.

Finally, it is necessary to take into account that security is another outstanding feature in SDWAN because it is present during connectivity, traffic management, additional security services, deployment, visibility and compliance with what is configured [13]. In addition, security is necessary for any company and from the point of view of service providers, security is an essential requirement to be able to implement their projects, although there are different architectures, designs and brands [14].

Based on what was read, it concludes that software-defined WAN networks represent a viable solution to address the difficulties that arise in a traditional WAN network. In addition, it allows to ensure the quality of service through efficient management of network resources, which allows improving network performance, availability and security. Another point to highlight is the reduction of costs in infrastructure and human resources, especially since this technology is simple to administer, which allows a staff with basic knowledge of networks to create offices and perform agile configurations, which shows us that the difficulty in managing networks is significantly reduced, making this type of technology become the next generation network.

\section{Methodology}

The methodology used in this research is Waterfall (see Fig. 1) because the research activities are distributed in phases which are developed sequentially. It is important to mention that a phase cannot be started if the previous one has not been completed. That is why the Scrum framework is not used [15] [16] [17] [18].

\section{A. Phase 1 - Start}

1) Project Scope: Functional requirements: It is necessary to keep in mind that the focus of this research is associated with

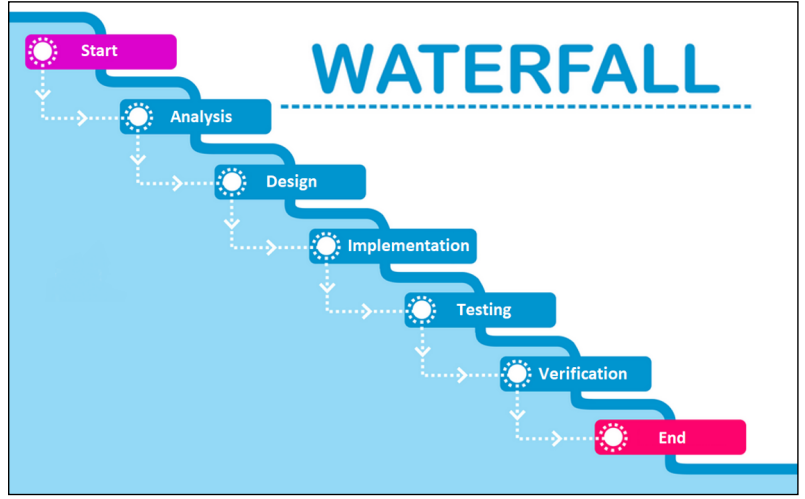

Fig. 1. Implementation Methodology.

the quantitative part, therefore, a laboratory will be executed that will provide results associated with parameters, for this it is necessary to cover certain technical requirements at the software and hardware level to be able to carry out the laboratory.

To perform the deployment for both the traditional environment and the SDWAN environment, PNETLAB tool will be used. This software is a virtual machine that can be provisioned on a VMWare Workstation. The advantage of this technology is that it is not necessary to invest to buy physical devices to be able to emulate the hardware and software.

The laboratory is going to use virtualized devices that are associated with one of the best-known brands in the network and communications environment. Cisco was the chosen brand because it its software can be executed in a virtualized environment and also its entire platform is supported and maintained, thus providing reliability for laboratory development. Table I and Table II specify hardware requirements for traditional WAN and SDWAN environments. The hardware and software requirements for the server are detailed in Tables III and IV.

TABLE I. HARDWARE REQUIREMENTS SDWAN

\begin{tabular}{|c|c|c|c|c|c|}
\hline Device & Quantity & CPU & Memory & Total CPU & Total Memory \\
\hline vBond & 1 & 1 & 1 & 1 & 1 \\
\hline vManage & 1 & 4 & 16 & 4 & 16 \\
\hline vSmart & 3 & 2 & 2 & 6 & 6 \\
\hline vEdge & 3 & 1 & 1 & 3 & 3 \\
\hline vIOS_L2 & 1 & 1 & 1 & 1 & 1 \\
\hline vIOS & 8 & 1 & 1 & 8 & 8 \\
\hline Computer & 4 & 2 & 8 & 8 & 24 \\
\hline TOTAL & 22 & & & 31 & 59 \\
\hline
\end{tabular}

TABLE II. HARDWARE REQUIREMENTS TRADITIONAL WAN

\begin{tabular}{|c|c|c|c|c|c|}
\hline Device & Quantity & CPU & Memory & Total CPU & Total Memory \\
\hline vIOS & 7 & 1 & 1 & 7 & 7 \\
\hline vIOS_L2 & 1 & 1 & 1 & 1 & 1 \\
\hline vNXOS & 2 & 1 & 8 & 2 & 16 \\
\hline Computer & 4 & 2 & 8 & 8 & 32 \\
\hline TOTAL & 14 & & & 18 & 56 \\
\hline
\end{tabular}

TABLE III. HARDWARE REQUIREMENTS FOR THE SERVER

\begin{tabular}{|c|c|c|c|c|}
\hline Device & CPU(Core) & CPU(vCore) & Memory(GB) & Storage(SSD/TB) \\
\hline Server & 16 & 32 & 128 & 1 \\
\hline
\end{tabular}


TABLE IV. SOFTWARE REQUIREMENTS For THE SERVER

\begin{tabular}{|c|c|c|}
\hline App & Version & Description \\
\hline Windows & 10 & Operative System \\
\hline VMWare Workstation & 16.1 .2 & Virtual Machine Hypervisor \\
\hline PNETLAB & 4.2 .9 & Simulator Tool \\
\hline vIOS & $15.6(2) \mathrm{T}$ & Virtual Router \\
\hline vIOS_L2 & $15.2(4.0 .55) \mathrm{E}$ & Virtual Switch Layer 3 \\
\hline vNXOS & 9.2 .2 & Virtual Switch Nexus \\
\hline vManage & 20.3 .2 .1 & Virtual Manager \\
\hline vSmart & 20.3 .2 & Virtual Route Processor \\
\hline vBond & 20.3 .2 & Virtual Orchestrator \\
\hline vEdge & 20.3 .2 & Virtual Edge Router \\
\hline
\end{tabular}

\section{2) Project Deliverables:}

- Testing scopes

- Work breakdown structure

- Topological diagrams

- Gantt diagram

- Polls

- Survey results

- Results of internet quotes

- Laboratory results

\section{B. Phase 2 - Analysis}

\section{1) Traditional Architecture:}

Traditional WAN technology is connection-oriented, meaning that its main purpose is to carry information from a source to a destination. In addition, the main weaknesses of the traditional WAN are its manual configuration, its reactive response to incidents, it is oriented to the use of physical components to function and it is not compatible with other programming tools.

Currently, the traditional WAN architecture can cover the different ways of getting from one point to another either by LTE, MPLS, internet or satellite, however, it does not provide dynamism when connecting a point of origin to destination.

Fig. 2 illustrates communication in a traditional WAN environment. It should be noted that communication in a company has the priority of using an MPLS network, in which the components are configured manually. For LTE, internet and satellite, private communications called VPNs are created, which remain manual settings.

2) SDWAN Architecture: For SDWAN, communication is not only based on communicating a point of origin to a destination, but also provides greater benefits such as the automation of configurations, virtual machines can be used and it is compatible with APIs, for this reason it is programmable. In addition, it uses a graphical interface to be able to visualize the health status and settings in a centralized way. Also, it is not oriented to the connection, but to the business need, for example, when a device has two links and one of them is congested, SDWAN has the ability to use a connection that is less congested.

Some of the strengths of SDWAN is that an additional layer of security can be added for information processing and the

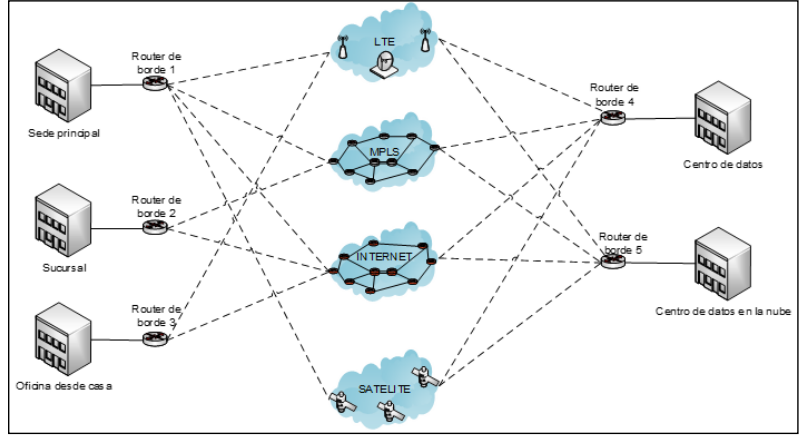

Fig. 2. Traditional WAN Architecture.

provisioning that is given to the equipment is done from a web interface, it is not necessary to configure the equipment onsite, it just requires access to internet and also it needs to be configured with a minimum administration parameters. In addition, it provides analytics on traffic use, applications, users and devices. This makes it a tool that gives companies visibility on bandwidth usage.

Fig. 3 illustrates the communication that occurs between vEdge devices, that are associated with a dynamic communication between any other, also, for each connection a secure and private tunnel is created while the communication is stablished. The only thing they need is to have ip connectivity between them. The intelligence at the routing level is provided by the vSmart controllers. In addition, you can see the communication between the different premises that, unlike the traditional WAN, is dynamic and provides flexibility when configuring because templates are used and are applied in an automated way.

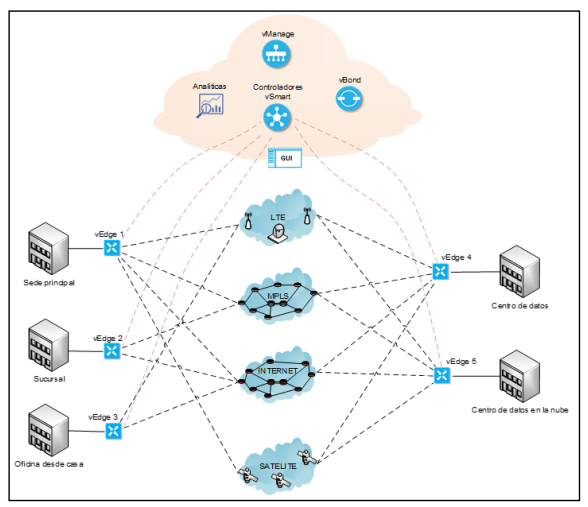

Fig. 3. Cisco SD WAN Architecture.

3) Risks: It is necessary to bear in mind that unexpected events can occur even in a controlled environment, in this case a virtual environment that is simulated by a tool. These risks can be found at the hardware level, software level, or even human error. The most important risks to consider for the development of the laboratory implementation are detailed below.

- $\quad$ Risk 1: Bugs

- Description: Error caused by SDWAN software code 
- Cause: Defective software

- Consequence: Malfunction in some of the SDWAN services

- Probability: Low

Impact: High

Risk Level: Considerable

Control: Use the latest recommended version

- $\quad$ Risk 2: Performance

- Description: Server saturation for virtual environment emulation

- Cause: Limited server resources

- Consequence: Slow use of virtualized computers services

- Probability: Low

- Impact: High

- Risk Level: Considerable

- Control: Run one virtual environment at a time

- Risk 3: Human error

- Description: Error in the configuration of virtualized machines

- Cause: Disorder in virtual machine configuration

- Consequence: Lack of connectivity or malfunction in virtualized communication equipment

○ Probability: Medium

- Impact: Medium

- Risk Level: Considerable

- Control: Review the equipment configurations so that a double check is performed

\section{Phase 3 - Design}

In this phase, a topology is created for traditional WAN and SDWAN environments. Each of the environments will have similar parts as their own technology. It is necessary to keep in mind that the topology for both is logical and physical and also it is oriented for a technical purpose.

A well known design was used in WAN Traditional topology; for SDWAN design MPLS and Internet must have at least a connection between them in order to communicate servers network to Internet interface for vedges.

1) Technical Scope for Laboratory: For MPLS and Internet dynamyc routing protocols were used. For practical purpose, OSPF was used.

2) WAN Traditional Topology : The connection must be made from Headqueaters to Data center using MPLS and Internet as backup only with MPLS's link is down (see Fig. 4).

3) SDWAN Topology : The connection must be made from Headqueaters to Data center using MPLS as a priority and Internet as backup with latency is high (see Fig. 5).

4) Work Breakdown Structure: These are the activities used for the laboratory (see Fig. 6).

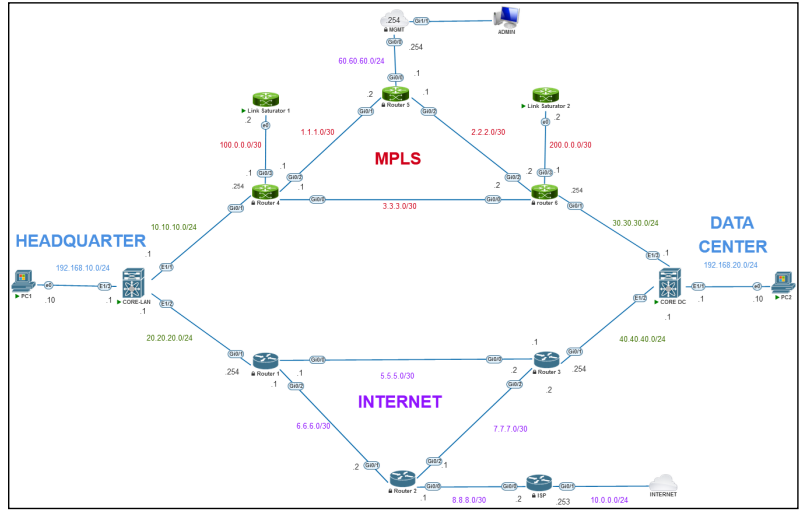

Fig. 4. WAN Topology.

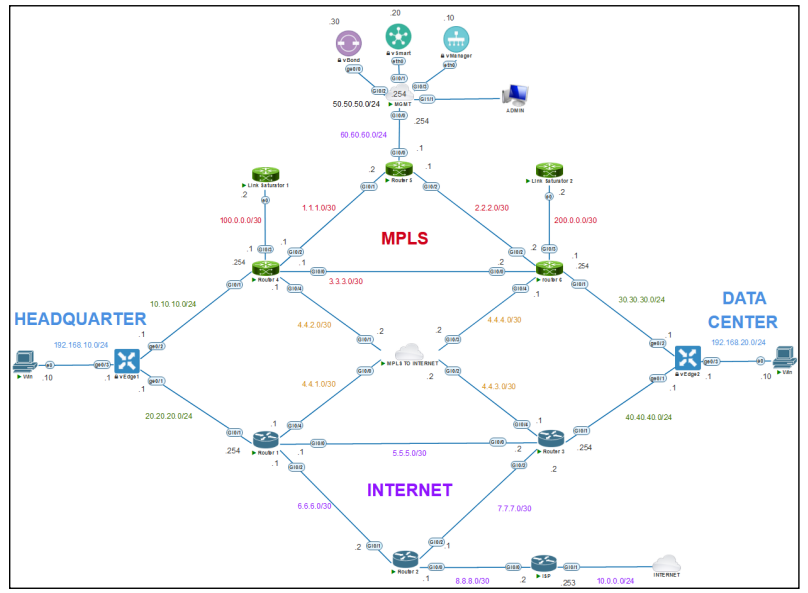

Fig. 5. SDWAN Topology.

\section{Phase 4 - Implementation}

In the next section shows aspects at the IP addressing level that will be used to run the laboratory and optimize communication between the different communication devices.

1) Traditional WAN Environment : The Table V consider the devices shown in Fig. 4.

2) SDWAN Environment : The Table VI consider the devices shown in Fig. 5.

\section{RESUlTS AND Discussions}

\section{A. Phase 5 - Testing}

1) Testing Traditional WAN: The tests are based on evaluating two scenarios, one without saturation and the other with saturation in order to evaluate the behavior of a traditional WAN network. The objective of these tests is to validate that the current technology is connection oriented and does not have an intelligence that adapts to changes in the network and can make routing decisions that benefit more stable connectivity in case of intermittences or saturation.

All tests performed consider the path to the MPLS as a routing priority because it is a dedicated network for the client, however, this network in many occasions in a real scenario is 


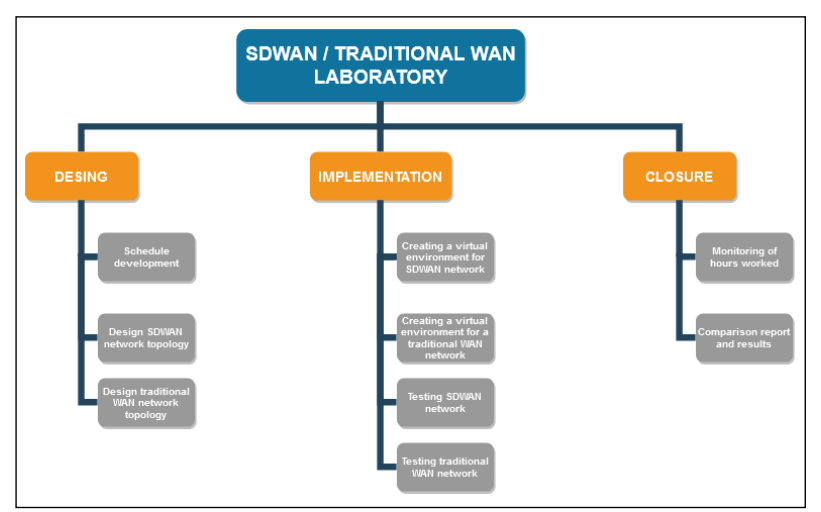

Fig. 6. Work Breakdown Structure.

TABLE V. TRADITIONAL WAN ENVIRONMENT IP ADDRESSING

\begin{tabular}{|c|c|c|c|}
\hline HOSTNAME & LOCATION & IP/MASK & GATEWAY \\
\hline Computer 1 & Headquarter & $192.168 .10 .10 / 24$ & 192.168 .10 .1 \\
\hline Computer 2 & Data Center & $192.168 .20 .10 / 24$ & 192.168 .20 .1 \\
\hline Link Saturator 1 & Mpls & $100.0 .0 .2 / 30$ & 100.0 .0 .1 \\
\hline Link Saturator 2 & Mpls & $200.0 .0 .2 / 30$ & 200.0 .0 .1 \\
\hline \multirow{3}{*}{ Router 1} & \multirow{3}{*}{ Mpls } & $5.5 .5 .1 / 30$ & \\
\hline & & $6.6 .6 .1 / 30$ & \\
\hline & & $20.20 .20 .254 / 24$ & \\
\hline \multirow{3}{*}{ Router 2} & \multirow{3}{*}{ Mpls } & 6.6.6.2/30 & \\
\hline & & $7.7 .7 .1 / 30$ & \\
\hline & & $8.8 .8 .1 / 30$ & \\
\hline \multirow{3}{*}{ Router 3} & \multirow{3}{*}{ Mpls } & $5.5 .5 .2 / 30$ & \\
\hline & & $7.7 .7 .2 / 30$ & \\
\hline & & $40.40 .40 .254 / 24$ & \\
\hline \multirow{4}{*}{ Router 4} & \multirow{4}{*}{ Internet } & $1.1 .1 .1 / 30$ & \\
\hline & & $3.3 .3 .1 / 30$ & \\
\hline & & $10.10 .10 .254 / 24$ & \\
\hline & & $100.0 .0 .1 / 30$ & \\
\hline \multirow{3}{*}{ Router 5} & \multirow{3}{*}{ Internet } & $1.1 .1 .2 / 30$ & \\
\hline & & $60.60 .60 .1 / 24$ & \\
\hline & & $2.2 .2 .1 / 30$ & \\
\hline \multirow{4}{*}{ Router 6} & \multirow{4}{*}{ Internet } & $2.2 .2 .2 / 30$ & \\
\hline & & $3.3 .3 .2 / 30$ & \\
\hline & & $30.30 .30 .254 / 24$ & \\
\hline & & $200.0 .0 .1 / 30$ & \\
\hline \multirow{3}{*}{ Core LAN } & \multirow{3}{*}{ Headquarter } & $10.10 .10 .1 / 24$ & \\
\hline & & $20.20 .20 .1 / 24$ & \\
\hline & & $192.168 .10 .1 / 24$ & \\
\hline \multirow{3}{*}{ Core DC } & \multirow{3}{*}{ Data Center } & $30.30 .30 .1 / 24$ & \\
\hline & & $40.40 .40 .1 / 24$ & \\
\hline & & $192.168 .20 .1 / 24$ & \\
\hline \multirow{2}{*}{ ISP } & \multirow{2}{*}{ Internet } & $8.8 .8 .2 / 30$ & \\
\hline & & $10.0 .0 .253 / 24$ & 10.0 .0 .1 \\
\hline INTERNET & Internet & $10.0 .0 .1 / 24$ & 192.168.1.1 \\
\hline ADMIN & Internet & $10.0 .0 .10 / 24$ & 10.0 .0 .1 \\
\hline \multirow{2}{*}{ MGMT } & \multirow{2}{*}{ Internet } & $50.50 .50 .254 / 24$ & \\
\hline & & $60.60 .60 .1 / 30$ & \\
\hline
\end{tabular}

also subject to congestion or falls, therefore, a situation will be created in which it is stressed or congested by a lot of traffic.

a) No link saturation : Fig. 7 and Fig. 8 shows the Netscantools application after having taken the capture of information for 100 seconds without saturation.

For these tests, ping, latency and jitter and traceability tests have been considered. These tests refer to the hops between devices from a point of origin to a destination. Likewise, Netscantools provides information both at a graphic and textual level, this is very important because it gives visibility of the times that exist while the information goes from one point to another (see Fig. 9).
TABLE VI. SDWAN ENVIRONMENT IP ADDRESSING

\begin{tabular}{|c|c|c|c|}
\hline HOSTNAME & LOCATION & IP/MASK & GATEWAY \\
\hline Computer 1 & Headquarter & $192.168 .10 .10 / 24$ & 192.168.10.1 \\
\hline Computer 2 & Data Center & $192.168 .20 .10 / 24$ & 192.168.20.1 \\
\hline Link Saturator 1 & Mpls & $100.0 .0 .2 / 30$ & 100.0 .0 .1 \\
\hline Link Saturator 2 & Mpls & $200.0 .0 .2 / 30$ & 200.0 .0 .1 \\
\hline \multirow{3}{*}{ Router 1} & \multirow{3}{*}{ Mpls } & $5.5 .5 .1 / 30$ & \\
\hline & & 6.6.6.1/30 & \\
\hline & & $20.20 .20 .254 / 24$ & \\
\hline \multirow{3}{*}{ Router 2} & \multirow{3}{*}{ Mpls } & 6.6.6.2/30 & \\
\hline & & $7.7 .7 .1 / 30$ & \\
\hline & & $8.8 .8 .1 / 30$ & \\
\hline \multirow{3}{*}{ Router 3} & \multirow{3}{*}{ Mpls } & $5.5 .5 .2 / 30$ & \\
\hline & & 7.7.7.2/30 & \\
\hline & & $40.40 .40 .254 / 24$ & \\
\hline \multirow{4}{*}{ Router 4} & \multirow{4}{*}{ Internet } & 1.1.1.1/30 & \\
\hline & & $3.3 .3 .1 / 30$ & \\
\hline & & $10.10 .10 .254 / 24$ & \\
\hline & & $100.0 .0 .1 / 30$ & \\
\hline \multirow{3}{*}{ Router 5} & \multirow{3}{*}{ Internet } & $1.1 .1 .2 / 30$ & \\
\hline & & $60.60 .60 .1 / 24$ & \\
\hline & & $2.2 .2 .1 / 30$ & \\
\hline \multirow{4}{*}{ Router 6} & \multirow{4}{*}{ Internet } & $2.2 .2 .2 / 30$ & \\
\hline & & $3.3 .3 .2 / 30$ & \\
\hline & & $30.30 .30 .254 / 24$ & \\
\hline & & $200.0 .0 .1 / 30$ & \\
\hline \multirow{3}{*}{ vEdge1 } & \multirow{3}{*}{ Headquarter } & $10.10 .10 .1 / 24$ & \\
\hline & & $20.20 .20 .1 / 24$ & \\
\hline & & $192.168 .10 .1 / 24$ & \\
\hline \multirow{3}{*}{ vEdge2 } & \multirow{3}{*}{ Data Center } & $30.30 .30 .1 / 24$ & \\
\hline & & $40.40 .40 .1 / 24$ & \\
\hline & & $192.168 .20 .1 / 24$ & \\
\hline vManage & Mpls & $50.50 .50 .10 / 24$ & 50.50 .50 .254 \\
\hline vSmart & Mpls & $50.50 .50 .20 / 24$ & 50.50 .50 .254 \\
\hline vBond & Mpls & $50.50 .50 .30 / 24$ & 50.50 .50 .254 \\
\hline \multirow{2}{*}{ ISP } & \multirow{2}{*}{ Internet } & $8.8 .8 .2 / 30$ & \\
\hline & & $10.0 .0 .253 / 24$ & 10.0 .0 .1 \\
\hline INTERNET & Internet & $10.0 .0 .1 / 24$ & 192.168.1.1 \\
\hline ADMIN & Internet & $\begin{array}{l}10.0 .0 .10 / 24 \\
\end{array}$ & $\begin{array}{l}10.0 .0 .1 \\
\end{array}$ \\
\hline \multirow{2}{*}{ MGMT } & \multirow{2}{*}{ Internet } & $50.50 .50 .254 / 24$ & \\
\hline & & $60.60 .60 .1 / 30$ & \\
\hline
\end{tabular}

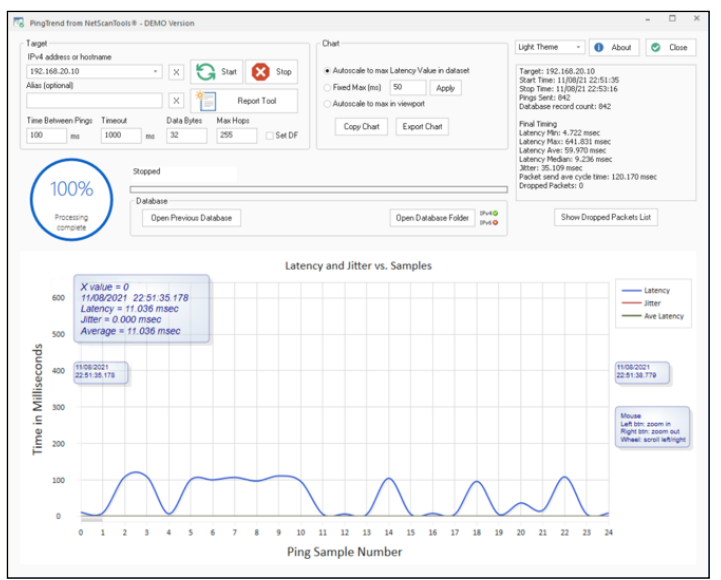

Fig. 7. Test Results without Saturation, no Link Saturation.

b) With link saturation: For this part, it was considered an additional program that generates traffic and allows to simulate congestion or saturation in the MPLS network. Fig. 10 show the program that was used to generate traffic on the network.

Fig. 11 and Fig. 12 show the results of the Netscantools application after running the test for 100 seconds with saturation. Fig. 13 shows the last traceroute of the tests. 


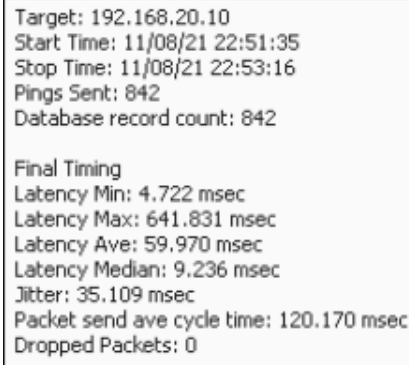

Fig. 8. Closeup of the Results, no Link Saturation.

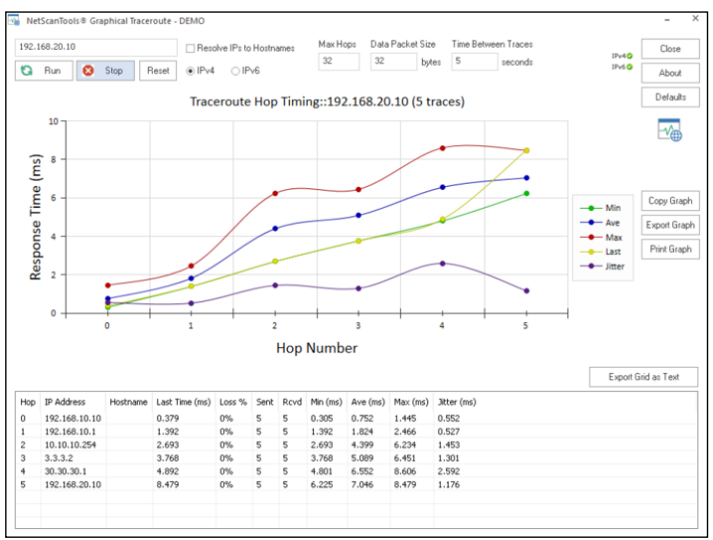

Fig. 9. Five Trace Information.

2) Testing SDWAN: In this case, two scenarios will also be evaluated, one without saturation and one with saturation in order to find. These tests will validate that the new softwaredefined network technology allows automatic changes in the network to make routing decisions that benefit more stable connectivity in case of intermittences or saturation of the links.

a) No link saturation : Fig. 14 shows the Netscantools application capture with the resulting information after running the test for 100 seconds without saturation.

Fig. 15 correspond to the graph obtained during the execution time of the test. The values obtained allow to validate that there are minimal changes in the communication speed. It is necessary to take into account that the times are considered in milliseconds.

As in the traditional WAN laboratory, traceability tests have also been considered, which refer to the number of computers through which the information passes from a point of origin to a destination. The tests will be executed in 5 consecutive times, leaving a lapse of 5 seconds between each one. Fig. 16 shows the last traceroute of the tests run.

b) With link saturation : Fig. 17 and Fig. 18 are the result after having run the tests for 100 seconds with saturation. Fig. 19 shows the last traceroute of the tests.

\section{3) Traditional WAN Lab Results :}

a) Latency: The following tables compare the results at the latency level obtained in the traces of the tests executed with and without saturation of the links. Table VII compares

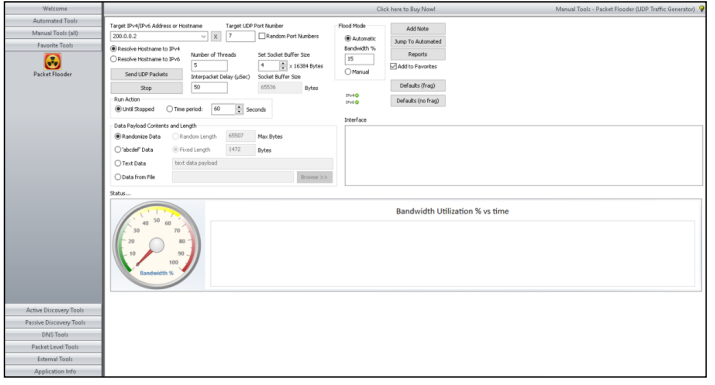

Fig. 10. Saturation Program Configuration.

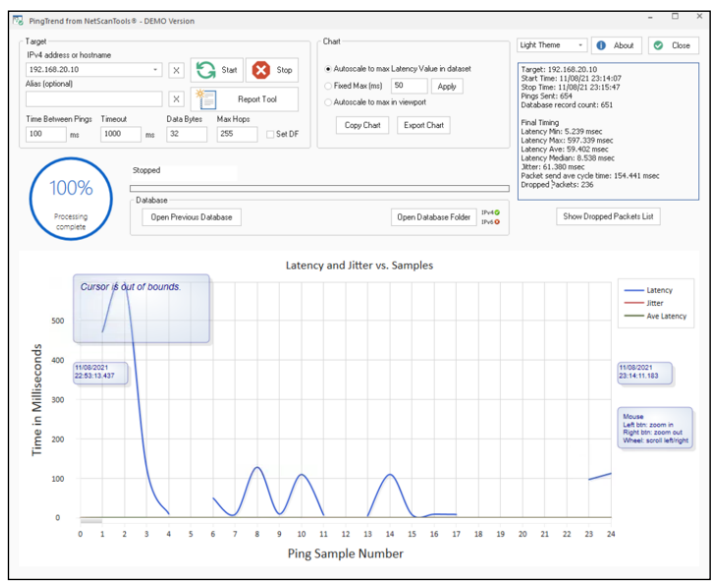

Fig. 11. Test Results of the Netscantools Application with Saturation.

latency level with and without saturation. Table VIII and Table IX show the minimum and maximum values obtained in the tests and Table $\mathrm{X}$ compares the average values.

TABLE VII. COMPARISON OF THE LEVEL OF LATENCY WITH AND WITHOUT SATURATION WAN

\begin{tabular}{|c|c|c|c|c|}
\hline Hop & IP & $\begin{array}{c}\text { Without Sat. } \\
(\mathrm{ms})\end{array}$ & $\begin{array}{c}\text { With Sat. } \\
(\mathrm{ms})\end{array}$ & $\begin{array}{c}\text { Difference } \\
(\mathrm{ms})\end{array}$ \\
\hline 0 & 192.168 .10 .10 & 0.379 & 0.281 & -0.10 \\
\hline 1 & 192.168 .10 .1 & 1.392 & 1.707 & 0.32 \\
\hline 2 & 10.10 .10 .254 & 2.693 & 790.459 & 787.77 \\
\hline 3 & 3.3 .3 .2 & 3.768 & 2540.401 & 2536.63 \\
\hline 4 & 30.30 .30 .1 & 4.892 & 5.298 & 0.41 \\
\hline 5 & 192.168 .20 .10 & 8.479 & 8.162 & -0.32 \\
\hline
\end{tabular}

TABLE VIII. COMPARISON OF MINIMUM WITHOUT AND WITHOUT SATURATION IN MILLISECONDS WAN

\begin{tabular}{|c|c|c|c|c|}
\hline Hop & IP & $\begin{array}{c}\text { Without Sat. } \\
\text { Min }(\mathrm{ms})\end{array}$ & $\begin{array}{c}\text { With Sat. } \\
\text { Min }(\mathrm{ms})\end{array}$ & $\begin{array}{c}\text { Difference } \\
(\mathrm{ms})\end{array}$ \\
\hline 0 & 192.168 .10 .10 & 0.305 & 0.281 & -0.02 \\
\hline 1 & 192.168 .10 .1 & 1.392 & 1.45 & 0.06 \\
\hline 2 & 10.10 .10 .254 & 2.693 & 648.543 & 645.85 \\
\hline 3 & 3.3 .3 .2 & 3.768 & 619.221 & 615.45 \\
\hline 4 & 30.30 .30 .1 & 4.801 & 5.298 & 0.50 \\
\hline 5 & 192.168 .20 .10 & 6.225 & 6.791 & 0.57 \\
\hline
\end{tabular}




\begin{tabular}{|l|}
\hline Target: 192.168 .20 .10 \\
Start Time: $11 / 08 / 21$ 23:14:07 \\
Stop Time: $11 / 08 / 21$ 23:15:47 \\
Pings Sent: 654 \\
Database record count: 651 \\
\\
Final Timing \\
Latency Min: $5.239 \mathrm{msec}$ \\
Latency Max: $597.339 \mathrm{msec}$ \\
Latency Ave: $59.402 \mathrm{msec}$ \\
Latency Median: $8.538 \mathrm{msec}$ \\
Jitter: $61.380 \mathrm{msec}$ \\
Packet send ave cycle time: $154.441 \mathrm{msec}$ \\
Dropped Packets: 236
\end{tabular}

Fig. 12. Closeup of the Results, with Link Saturation.

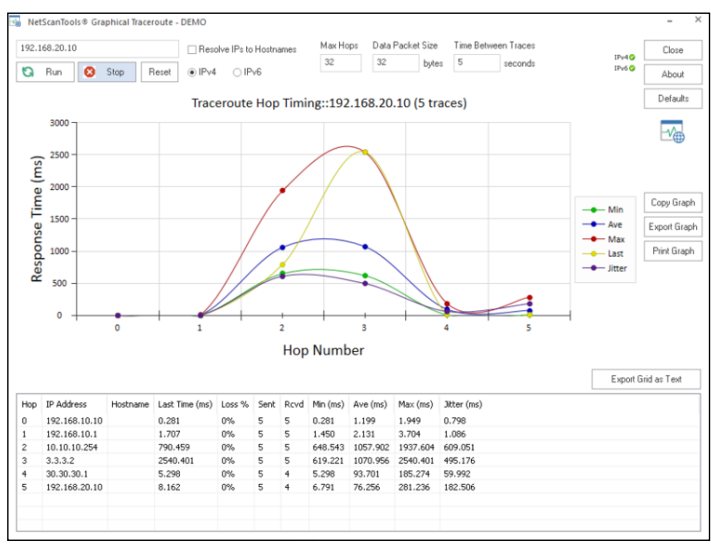

Fig. 13. Fifth Trace Graph of the Test.

TABLE IX. COMPARISON OF MAXIMUM WITHOUT AND WITHOUT SATURATION IN MILLISECONDS WAN

\begin{tabular}{|c|c|c|c|c|}
\hline Hop & IP & $\begin{array}{c}\text { Without } \\
\text { Sat. } \\
\text { Max (ms) }\end{array}$ & $\begin{array}{c}\text { With } \\
\text { Sat. } \\
\text { Maxi (ms) }\end{array}$ & $\begin{array}{c}\text { Difference } \\
(\mathrm{ms})\end{array}$ \\
\hline 0 & 192.168 .10 .10 & 1.445 & 1.949 & 0.50 \\
\hline 1 & 192.168 .10 .1 & 2.466 & 3.704 & 1.24 \\
\hline 2 & 10.10 .10 .254 & 6.234 & 1937.604 & 1931.37 \\
\hline 3 & 3.3 .3 .2 & 6.451 & 2540.401 & 2533.95 \\
\hline 4 & 30.30 .30 .1 & 8.606 & 185.274 & 176.67 \\
\hline 5 & 192.168 .20 .10 & 8.479 & 281.236 & 272.76 \\
\hline
\end{tabular}

TABLE X. COMPARISON OF AVERAGE WITHOUT AND WITHOUT SATURATION IN MILLISECONDS WAN

\begin{tabular}{|c|c|c|c|c|}
\hline Hop & IP & $\begin{array}{c}\text { Without } \\
\text { Sat. } \\
\text { Average }(\mathrm{ms})\end{array}$ & $\begin{array}{c}\text { With } \\
\text { Sat. } \\
\text { Average }(\mathrm{ms})\end{array}$ & $\begin{array}{c}\text { Difference } \\
(\mathrm{ms})\end{array}$ \\
\hline 0 & 192.168 .10 .10 & 0.752 & 1.199 & 0.45 \\
\hline 1 & 192.168 .10 .1 & 1.824 & 2.131 & 0.31 \\
\hline 2 & 10.10 .10 .254 & 4.399 & 1057.902 & 1053.50 \\
\hline 3 & 3.3 .3 .2 & 5.089 & 1070.956 & 1065.87 \\
\hline 4 & 30.30 .30 .1 & 6.552 & 93.701 & 87.15 \\
\hline 5 & 192.168 .20 .10 & 7.046 & 76.256 & 69.21 \\
\hline
\end{tabular}

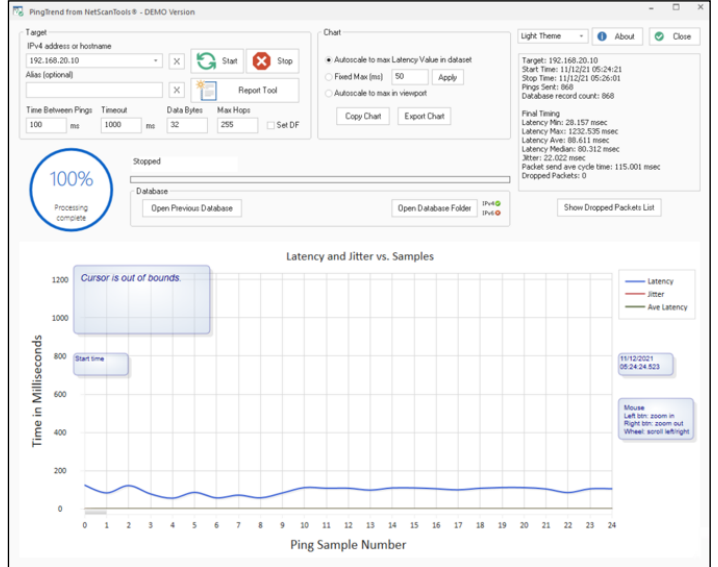

Fig. 14. Netscantools Application Capture with the Resulting Information.

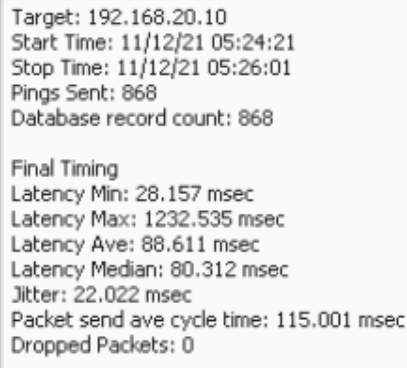

Fig. 15. Graph Obtained During the Execution Time of the Test.

TABLE XI. COMPARISON OF JITTER WITHOUT AND WITH SATURATION IN MILLISECONDS WAN ENVIRONMENT

\begin{tabular}{|c|c|c|c|c|}
\hline Hop & IP & $\begin{array}{c}\text { Without } \\
\text { Sat } \\
\text { Jitter }(\mathrm{ms})\end{array}$ & $\begin{array}{c}\text { With } \\
\text { Sat. } \\
\text { Jitter(ms) }\end{array}$ & $\begin{array}{c}\text { Difference } \\
(\mathrm{ms})\end{array}$ \\
\hline 0 & 192.168 .10 .10 & 0.552 & 0.798 & 0.25 \\
\hline 1 & 192.168 .10 .1 & 0.527 & 1.086 & 0.56 \\
\hline 2 & 10.10 .10 .254 & 1.453 & 609.051 & 607.60 \\
\hline 3 & 3.3 .3 .2 & 1.301 & 495.176 & 493.88 \\
\hline 4 & 30.30 .30 .1 & 2.592 & 59.992 & 57.40 \\
\hline 5 & 192.168 .20 .10 & 1.176 & 182.506 & 181.33 \\
\hline
\end{tabular}

b) Jitter: Table XI compares the results of jitter level obtained in the traces of the tests with and without saturation of the links

\section{4) SDWAN Lab Results:}

a) Latency: The following tables compare the results at the latency level obtained in the traces of the tests executed with and without saturation of the links. Table XII compares latency level with and without saturation. Table XIII and Table XIV show the minimum and maximum values obtained in the tests and Table XV compares the average values. 


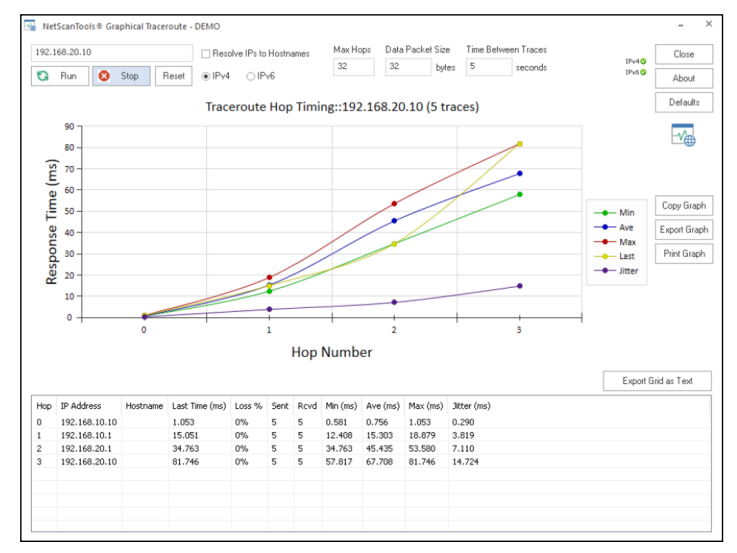

Fig. 16. Last Trace Graph of the Test Run.

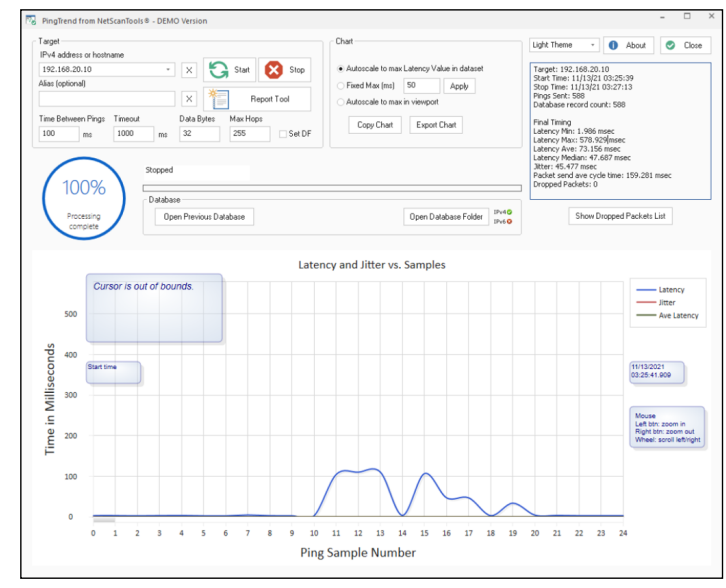

Fig. 17. Test Results with Saturation.

TABLE XII. TRACE COMPARISON WITHOUT AND WITH SATURATION IN MILLISECONDS SDWAN

\begin{tabular}{|c|c|c|c|c|}
\hline Hop & IP & $\begin{array}{c}\text { Final } \\
\text { Trace } \\
(\mathrm{ms})\end{array}$ & $\begin{array}{c}\text { Final } \\
\text { Trace } \\
(\mathrm{ms})\end{array}$ & $\begin{array}{c}\text { Difference } \\
(\mathrm{ms})\end{array}$ \\
\hline 0 & 192.168 .10 .10 & 0.575 & 1.053 & 0.48 \\
\hline 1 & 192.168 .10 .1 & 1.702 & 15.051 & 13.35 \\
\hline 2 & 192.168 .20 .1 & 4.111 & 34.763 & 30.65 \\
\hline 3 & 192.168 .20 .10 & 3.65 & 81.746 & 78.10 \\
\hline
\end{tabular}

TABLE XIII. COMPARISON OF MINIMUM WITHOUT AND WITHOUT SATURATION IN MILLISECONDS SDWAN

\begin{tabular}{|c|c|c|c|}
\hline Hop & IP & $\begin{array}{c}\text { Without Sat. } \\
\text { Minimum } \\
(\mathrm{ms})\end{array}$ & $\begin{array}{c}\text { With Sat. } \\
\text { Min } \\
(\mathrm{ms})\end{array}$ \\
\hline 0 & 192.168 .10 .10 & 0.289 & 0.581 \\
\hline 1 & 192.168 .10 .1 & 1.402 & 12.408 \\
\hline 2 & 192.168 .20 .1 & 3.041 & 34.763 \\
\hline 3 & 192.168 .20 .10 & 3.082 & 57.817 \\
\hline
\end{tabular}

\begin{tabular}{l}
\hline Target: 192.168 .20 .10 \\
Start Time: $11 / 13 / 21$ 03:25:39 \\
Stop Time: $11 / 13 / 2103: 27: 13$ \\
Pings Sent: 588 \\
Database record count: 588 \\
Final Timing \\
Latency Min: $1.986 \mathrm{msec}$ \\
Latency Max: $578.929 \mid \mathrm{msec}$ \\
Latency Ave: $73.156 \mathrm{msec}$ \\
Latency Median: $47.687 \mathrm{msec}$ \\
Jitter: 45.477 msec \\
Packet send ave cycle time: $159.281 \mathrm{msec}$ \\
Dropped Packets: 0 \\
\hline \hline
\end{tabular}

Fig. 18. Ping Test Graph at the Start of the Test.

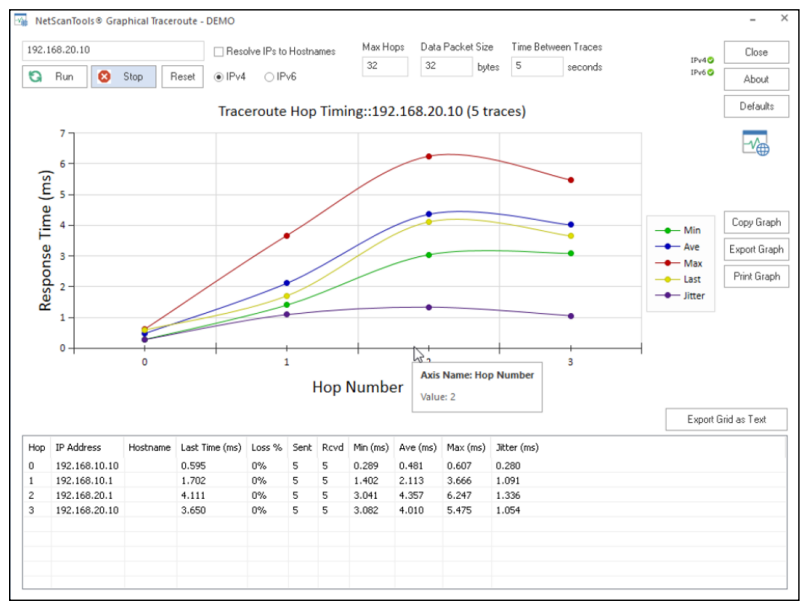

Fig. 19. Last Traceroute of the Test.

TABLE XIV. COMPARISON OF MAXIMUM WITHOUT AND WITHOUT SATURATION IN MILLISECONDS SDWAN

\begin{tabular}{|c|c|c|c|c|}
\hline Hop & IP & $\begin{array}{c}\text { Without Sat. } \\
\text { Maximum } \\
(\mathrm{ms})\end{array}$ & $\begin{array}{c}\text { With Sat. } \\
\text { Maximum } \\
(\mathrm{ms})\end{array}$ & $\begin{array}{c}\text { Difference } \\
(\mathrm{ms})\end{array}$ \\
\hline 0 & 192.168 .10 .10 & 0.607 & 1.053 & 0.45 \\
\hline 1 & 192.168 .10 .1 & 3.666 & 18.879 & 15.21 \\
\hline 2 & 192.168 .20 .1 & 6.247 & 53.58 & 47.33 \\
\hline 3 & 192.168 .20 .10 & 5.475 & 81.746 & 76.27 \\
\hline
\end{tabular}

TABLE XV. COMPARISON OF AVERAGE WITHOUT AND Without SATURATION IN MILLISECONDS SDWAN

\begin{tabular}{|c|c|c|c|c|}
\hline Hop & IP & $\begin{array}{c}\text { Without Sat. } \\
\text { Average } \\
(\mathrm{ms})\end{array}$ & $\begin{array}{c}\text { With Sat. } \\
\text { Average } \\
(\mathrm{ms})\end{array}$ & $\begin{array}{c}\text { Difference } \\
(\mathrm{ms})\end{array}$ \\
\hline 0 & 192.168 .10 .10 & 0.481 & 0.756 & 0.28 \\
\hline 1 & 192.168 .10 .1 & 2.113 & 15.303 & 13.19 \\
\hline 2 & 192.168 .20 .1 & 4.57 & 45.435 & 40.87 \\
\hline 3 & 192.168 .20 .10 & 4.01 & 67.708 & 63.70 \\
\hline
\end{tabular}

b) Jitter: Table XVI compares the results of jitter level obtained in the traces of the tests with and without saturation of the links. 
TABLE XVI. COMPARISON OF JITTER WITHOUT AND WITH SATURATION IN MiLLiseconds SDWAN EnVIRONMENT

\begin{tabular}{|c|c|c|c|c|}
\hline Hop & IP & $\begin{array}{c}\text { Without } \\
\text { Sat } \\
\text { Jitter (ms) }\end{array}$ & $\begin{array}{c}\text { With } \\
\text { Sat. } \\
\text { Jitter(ms) }\end{array}$ & $\begin{array}{c}\text { Difference } \\
(\mathrm{ms})\end{array}$ \\
\hline 0 & 192.168 .10 .10 & 0.28 & 0.29 & 0.01 \\
\hline 1 & 192.168 .10 .1 & 1.091 & 3.819 & 2.73 \\
\hline 2 & 192.168 .20 .1 & 1.336 & 7.11 & 5.77 \\
\hline 3 & 192.168 .20 .10 & 1.054 & 14.724 & 13.67 \\
\hline
\end{tabular}

\section{B. Phase 6 - Evaluation}

1) Technical Comparison: In the next section, the following tables represent the crossing of information between saturation tests of both technologies, also there is a difference in the number of hops, in SDWAN there a less because the connection is by tunneling.

a) Design: A big difference between both designs is that SDWAN needs to have servers hosted and Internet and MPLS must have a connection in order to get those servers. In the other hand, for traditional WAN, since each device will be managed independently.

b) Configuration: The configuration in the initial phase for SDWAN is executed by command line because it is necessary to assign some parameters for its registration and synchronization with the solution servers. The rest of the configurations are made from a graphical interface. In the other hand, for traditional WAN, $100 \%$ of the configurations are made in command lines and additionally the validations are done in the same way. It is important to mention that this can be done initially onsite and then it could be managed remotely.

c) Latency: In the results, it can be seen that for traditional WAN technology there is a higher rate of slowness in the response when there is saturation, whereas for SDWAN there is a certain increase in latency, but it is not so considerable (see Table XVII). The important thing is that the connectivity service in general would not be affected and users could perceive a certain slowness, but not disconnections that is a great benefit for SDWAN.

TABLE XVII. LATENCY COMPARISON BETWEEN SDWAN AND TRADITIONAL WAN

\begin{tabular}{|c|c|c|c|c|c|}
\cline { 4 - 6 } \multicolumn{1}{c|}{} & $\begin{array}{c}\text { WIthout } \\
\text { Saturation }\end{array}$ & $\begin{array}{c}\text { With } \\
\text { Saturation }\end{array}$ & \\
\cline { 2 - 6 } \multicolumn{1}{c|}{} & Hop & IP & $\begin{array}{c}\text { Final trace } \\
(\mathrm{ms})\end{array}$ & $\begin{array}{c}\text { Final trace } \\
(\mathrm{ms})\end{array}$ & $\begin{array}{c}\text { Difference } \\
(\mathrm{ms})\end{array}$ \\
\hline \multirow{4}{*}{$\begin{array}{c}\text { Traditional } \\
\text { WAN }\end{array}$} & 0 & 192.168 .10 .10 & 0.379 & 0.281 & -0.10 \\
\cline { 2 - 6 } & 1 & 192.168 .10 .1 & 1.392 & 1.707 & 0.32 \\
\cline { 2 - 6 } & 2 & 10.10 .10 .254 & 2.693 & 790.459 & 787.77 \\
\cline { 2 - 6 } & 3 & 3.3 .3 .2 & 3.768 & 2540.401 & 2536.63 \\
\cline { 2 - 6 } & 5 & 30.30 .30 .1 & 4.892 & 5.298 & 0.41 \\
\hline \multirow{4}{*}{ SDWAN } & 192.168 .20 .10 & 8.479 & 8.162 & -0.32 \\
\cline { 2 - 6 } & 1 & 192.168 .10 .10 & 0.575 & 1.053 & 0.48 \\
\cline { 2 - 6 } & 2 & 192.168 .10 .1 & 1.702 & 15.051 & 13.35 \\
\cline { 2 - 6 } & 3 & 192.168 .20 .1 & 4.111 & 34.763 & 30.65 \\
\hline
\end{tabular}

d) Jitter: SDWAN has better values because it can switch links in a smart way and this allows the communication to have better performance and quality (see Table XVIII).
TABLE XVIII. JITTER COMPARISON BETWEEN SDWAN AND TRADITIONAL WAN

\begin{tabular}{|c|c|c|c|c|c|}
\cline { 3 - 6 } \multicolumn{2}{c|}{} & $\begin{array}{c}\text { WIthout } \\
\text { Saturation }\end{array}$ & $\begin{array}{c}\text { With } \\
\text { Saturation }\end{array}$ & \\
\cline { 2 - 6 } \multicolumn{1}{c|}{} & Hop & IP & $\begin{array}{c}\text { Jitter } \\
(\mathrm{ms})\end{array}$ & $\begin{array}{c}\text { Jitter } \\
(\mathrm{ms})\end{array}$ & $\begin{array}{c}\text { Difference } \\
(\mathrm{ms})\end{array}$ \\
\hline \multirow{4}{*}{$\begin{array}{c}\text { Traditional } \\
\text { WAN }\end{array}$} & 0 & 192.168 .10 .10 & 0.552 & 0.798 & 0.25 \\
\cline { 2 - 6 } & 1 & 192.168 .10 .1 & 0.527 & 1.086 & 0.56 \\
\cline { 2 - 6 } & 2 & 10.10 .10 .254 & 1.453 & 609.051 & 607.60 \\
\cline { 2 - 6 } & 3 & 3.3 .3 .2 & 1.301 & 495.176 & 493.88 \\
\cline { 2 - 6 } & 4 & 30.30 .30 .1 & 2.592 & 59.992 & 57.40 \\
\cline { 2 - 6 } & 5 & 192.168 .20 .10 & 1.176 & 182.506 & 181.33 \\
\hline \multirow{4}{*}{ SDWAN } & 0 & 192.168 .10 .10 & 0.28 & 0.29 & 0.01 \\
\cline { 2 - 6 } & 2 & 192.168 .10 .1 & 1.091 & 3.819 & 2.73 \\
\cline { 2 - 6 } & 3 & 192.168 .20 .1 & 1.336 & 7.11 & 5.77 \\
\cline { 2 - 6 } & & 192.168 .20 .10 & 1.054 & 14.724 & 13.67 \\
\hline
\end{tabular}

\section{COnClusions AND Future WORK}

SDWAN is a technology that improves capabilities and characteristics over traditional technology in different aspects such as automatization, hardware agnostic , programmable, web interface interaction, business-oriented, cloud-based, secure, scalable and provides analytics. SDWAN networks allow branch offices to have greater communication availability with data centers by prioritizing critical traffic using different types of parameters such as delay or jitter. The result of the tests executed in a virtual environment validates the great benefits at the level of agility and scalability that the solution brings because it was shown that SDWAN was much more effective than the traditional WAN to continue communication despite the fact that the network was congested. It is concluded that SDWAN offers many technical benefits that in the end of the day will keep smart communications and save cost for companies. The next step is to investigate about the return on investment and comparison between the different SDWAN brands that exist in the market so that it can enrich the research presented in this document.

\section{REFERENCES}

[1] S. Badotra, S. N. Panda et al., "A survey on software defined wide area network," International Journal of Applied Science and Engineering, vol. 17, no. 1, pp. 59-73, 2020.

[2] X. Wu, K. Lu, and G. Zhu, "A survey on software-defined wide area networks." J. Commun., vol. 13, no. 5, pp. 253-258, 2018.

[3] S. Mohmmad, D. Ramesh, S. Pasha, K. Shankar et al., "Research on new network architecture through sd-wan," International Journal of Innovative Technology and Exploring Engineering, vol. 8, no. 6 Special Issue 4, pp. 483-490, 2019.

[4] Z. Yang, Y. Cui, B. Li, Y. Liu, and Y. Xu, "Software-defined wide area network (sd-wan): Architecture, advances and opportunities," in 2019 28th International Conference on Computer Communication and Networks (ICCCN). IEEE, 2019, pp. 1-9.

[5] R. E. Mora-Huiracocha, P. L. Gallegos-Segovia, P. E. Vintimilla-Tapia, J. F. Bravo-Torres, E. J. Cedillo-Elias, and V. M. Larios-Rosillo, "Implementation of a sd-wan for the interconnection of two software defined data centers," in 2019 IEEE Colombian Conference on Communications and Computing (COLCOM). IEEE, 2019, pp. 1-6.

[6] I. Šeremet and S. Čaušević, "Advancing ip/impls with software defined network in wide area network," in 2019 International Workshop on Fiber Optics in Access Networks (FOAN). IEEE, 2019, pp. 56-61.

[7] S. Troia, L. M. M. Zorello, A. J. Maralit, and G. Maier, "Sd-wan: an open-source implementation for enterprise networking services," in 2020 22nd International Conference on Transparent Optical Networks (ICTON). IEEE, 2020, pp. 1-4.

[8] H. Zhang, Y. Wang, X. Qi, W. Xu, T. Peng, and S. Liu, "Demo abstract: An intent solver for enabling intent-based sdn," in 2017 IEEE Conference on Computer Communications Workshops (INFOCOM WKSHPS). IEEE, 2017, pp. 968-969. 
[9] S. Rajagopalan, "An overview of sd-wan load balancing for wan connections," in 2020 4th International Conference on Electronics, Communication and Aerospace Technology (ICECA). IEEE, 2020, pp. $1-4$.

[10] P. Segeč, M. Moravčik, J. Uratmová, J. Papán, and O. Yeremenko, "Sdwan-architecture, functions and benefits," in 2020 18th International Conference on Emerging eLearning Technologies and Applications (ICETA). IEEE, 2020, pp. 593-599.

[11] S. Andromeda and D. Gunawan, "Techno-economic analysis from implementing sd-wan with 4g/lte, a case study in xyz company," in 2020 International Seminar on Intelligent Technology and Its Applications (ISITIA). IEEE, 2020, pp. 345-351.

[12] S. Sinha, R. Chowdhury, A. Das, and A. Ghosh, "Prospective sdwan shift: Newfangled indispensable industry driver," in Soft Computing Techniques and Applications. Springer, 2021, pp. 255-261.

[13] M. Wood, "Top requirements on the sd-wan security checklist," Network Security, vol. 2017, no. 7, pp. 9-11, 2017.

[14] _ "How to make sd-wan secure," Network Security, vol. 2017, no. 1, pp. 12-14, 2017.

[15] A. Carrion-Silva, C. Diaz-Nunez, and L. Andrade-Arenas, "Admission exam web application prototype for blind people at the university of sciences and humanities," International Journal of Advanced Computer Science and Applications, vol. 11, no. 12, 2020. [Online]. Available: http://dx.doi.org/10.14569/IJACSA.2020.0111246

[16] R. Arias-Marreros, K. Nalvarte-Dionisio, and L. Andrade-Arenas, "Design of a mobile application for the learning of people with down syndrome through interactive games," International Journal of Advanced Computer Science and Applications, vol. 11, no. 11, 2020. [Online]. Available: http://dx.doi.org/10.14569/IJACSA.2020.0111187

[17] A. Tupia-Astoray and L. Andrade-Arenas, "Implementation of an e-commerce system for the automation and improvement of commercial management at a business level," International Journal of Advanced Computer Science and Applications, vol. 12, no. 1, 2021. [Online]. Available: http://dx.doi.org/10.14569/IJACSA.2021.0120177

[18] V. Gomero-Fanny, A. R. Bengy, and L. Andrade-Arenas, "Prototype of web system for organizations dedicated to e-commerce under the scrum methodology," International Journal of Advanced Computer Science and Applications, vol. 12, no. 1, 2021. [Online]. Available: http://dx.doi.org/10.14569/IJACSA.2021.0120152 